\title{
Baffo secondo Apollinaire Una variante dell'erotomania apollinairiana
}

\section{Alessandro Metlica}

\section{(2) OpenEdition}

1 Journals

\section{Edizione digitale}

URL: http://journals.openedition.org/studifrancesi/6157

DOI: 10.4000/studifrancesi.6157

ISSN: 2421-5856

\section{Editore}

Rosenberg \& Sellier

\section{Edizione cartacea}

Data di pubblicazione: 1 novembre 2010

Paginazione: 456-467

ISSN: 0039-2944

\section{Notizia bibliografica digitale}

Alessandro Metlica, «Baffo secondo Apollinaire Una variante dell'erotomania apollinairiana», Studi Francesi [Online], 162 (LIV | III) | 2010, online dal 30 novembre 2015, consultato il 07 janvier 2021. URL: http://journals.openedition.org/studifrancesi/6157 ; DOl: https://doi.org/ERREUR PDO dans / localdata/www-bin/Core/Core/Db/Db.class.php L.34 : SQLSTATE[HY000] [2006] MySQL server has gone away

\section{(c)}

Studi Francesi è distribuita con Licenza Creative Commons Attribuzione - Non commerciale - Non opere derivate 4.0 Internazionale. 


\section{Baffo secondo Apollinaire Una variante dell'erotomania apollinairiana}

Noi pensammo dunque che il miglior mezzo per rompere la nefasta fama che toglie dalla libera circolazione queste opere belle e meritevoli di essere lette da tutti, è il divulgarle, non più sotto il velo del libro clandestino ma nella veste chiara e nobile del libro classico.

Così, nella breve nota introduttiva alla sua traduzione (1937) delle Vies des dames galantes ${ }^{1}$, Alberto Savinio giustificava la sua operazione di recupero nei confronti di un autore poco ortodosso come Pierre de Bourdeille, signore di Brantôme (1540$1614)^{2}$. Si badi bene: non mera curiosità bibliografica, prelibatezza per pochi e scelti palati, ma «libro classico» di valore assoluto, condannato alla clandestinità soltanto da un certo «pregiudizio puritano» ${ }^{3}$. Stando a giudicare da quanto questo saggio prenderà in esame, viene da pensare che anche per questo aspetto, certamente secondario, della sua figura intellettuale Savinio dovesse più di qualcosa al magistero di Apollinaire. Ne dà un'indiretta conferma Addio al poeta, uno dei racconti dell'Achille innamorato saviniano ${ }^{4}$ : Apollinaire vi viene rappresentato mentre, dando notizia a Max Jacob e al giovane De Chirico della sua partenza in guerra come volontario, traccia distrattamente su un foglio, quasi a bilanciare la gravità della dichiarazione, una quartina erotico-mitologica: «Le cul / D’Omphale / Vaincu / S'affale»5.

La passione del poeta francese per la letteratura di second rayon è cosa nota. Al momento di stendere il catalogo della sua biblioteca, Michel Décaudin e l'erede della collezione, Gilbert Boudar, non si stupivano di rintracciarvi una vasta serie di «publications anciennes, éditions déjà rares de Liseux, aussi bien que médiocres brochures clandestines $»^{6}$. È noto altresì che una larga fetta della produzione in prosa di Apollinaire si ispira ai moduli della letteratura "bassa" di consumo, e in particolare a quelli del romanzo storico-licenzioso: un corpus eterogeneo che la critica ha raramente indagato nel suo complesso ${ }^{7}$, specie in ragione della sua scarsa qualità. Mi riferisco

(1) BRANTÔME, Le dame galanti, traduzione di Alberto SAvinio, Milano, Adelphi, 1994, p. 10.

(2) L'opera di Brantôme venne peraltro ristampata, dopo la morte di Apollinaire, dallo stesso Georges Briffaut: P. BourdeIlle, Les dames galantes, édition publiée avec des notes et éclaircissements de Raoul VÈzE et des illustrations originales de Joseph HÉmARD, 2 voll., Paris, 1930-1931.

(3) BRAntôme, Le dame galanti, cit., p. 10.

(4) Cfr. A. SAVINIO, Casa «la Vita» e altri racconti, a cura di A. Tinterri e P. Italia, Milano, Adelphi, 1999 , pp. 38-40. Il racconto mette in scena l'ultimo incontro tra Savinio e Apollinaire; prima di trovare posto nell'Achille innamorato, fu pubblicato su «Nuovo Paese» il 6 marzo 1923 e, in francese, sull'«Esprit nouveau» del 26 ottobre 1924.

(5) Il sonetto, di cui i versi citati costituiscono la prima quartina, passerà poi ne L'Euvre libertine des poètes du XIX siècle («Les Maîtres de l'Amour», 1910), dove sarà attribuito ad un sedicente Abbé de Thélème, e di lì (o viceversa) nelle Onze mille verges. Per aver recitato questa e un'altra simile quelconquerie, il principe Vibescu vi verrà acclamato «poète archidivin» (G. ApolLINAIRE, Les onze mille verges ou les amours d'un Hospodar, préface de Michel DÉCAUDIN, Paris, Jean-Jacques Pauvert, 1973, p. 96).

(6) Catalogue de la bibliothèque de Guillaume Apollinaire, établi par Gilbert BOUDAR avec la collaboration de Michel DÉCAUDIN, Paris, Éditions du Centre National de la Recherche Scientifique, 1983, vol. I, p. 14.

(7) Il primo studio organico sull'argomento, salvo errore, è quello di D. Delbreil, Apollinaire et ses récits, Fasano, Schena, 1999. 
a libri scollacciati come La Rome des Borgia o La fin de Babylone, che nei primi anni dieci passavano agilmente per opere di Apollinaire e in buona parte, probabilmente, lo erano ${ }^{8}$. Anche Les trois Don Juan (1914), il terzo romanzo firmato da Apollinaire per «L'histoire romanesque» dei Briffaut, mirava soprattutto a solleticare nei lettori il gusto del proibito, benché il soggetto tirato in ballo promettesse qualcosa di più che una semplice riscrittura del mito in salsa avventurosa.

Il fatto è che l'operazione letteraria compiuta da Apollinaire per questi romanzi si limita ad un "copia e incolla" dalle fonti più disparate: si spazia da Erodoto alla Bibbia, ai compendi di magia esotica per i primi due testi; da Molière a Mérimée, dal Don Juan di Byron al romanzo picaresco d'area spagnola per il terzo, che sintetizza al meglio quel «mélange de plaisanterie et d'érudition»" che Apollinaire aveva in mente per simili pubblicazioni. Inoltre la facilità di scrittura sfoggiata in questi casi dal poeta, vicina a sfociare in vera e propria grafomania, non era estranea, come è facile intuire, a esigenze di schietta natura economica.

D'altronde si rifà agli stessi criteri anche il caso, certo più interessante, dei due romanzi propriamente pornografici di Apollinaire, Les exploits d'un jeune Don Juan e Les onze mille verges ${ }^{10}$. Il primo, in particolare, non è altro che la traduzione di un volume tedesco, uscito alla macchia e, a dirla tutta, di dubbia qualità: Kinder-Geilheit oder Geständnisse eines Knaben (La lascivia infantile o le confessioni di un fanciullo), pubblicato a Berlino nel $1891^{11}$. Ciò non toglie che si tratti, con ogni probabilità - specie se si prescinde dalla lunga gestazione dell'Hérésiarque et $C^{i e}$ e del Poète assassiné, che restano comunque raccolte di racconti - del primo romanzo di Apollinaire, e che decisamente apollinairiano sia il ritmo della narrazione, allegro e sostenuto, così come il taglio delle scene, fatto di accelerazioni, salti temporali e imprevedibili dissonanze narrative ${ }^{12}$. La stessa pratica della riscrittura non è priva di ripercussioni teoriche, visto che in questi romanzi "a cassetti" il sovvertimento dei codici, l'irrisione del genere letterario acquistano un significato autonomo di provocazione culturale ${ }^{13}$.

Questi temi emergono pienamente nel capolavoro dell'Apollinaire erotico, Les onze mille verges. Il folle pellegrinaggio, tra il picaresco e il sadiano, del principe Vibescu - che lascia Bucarest per Parigi, ritorna in patria a bordo dell'Orient Express, viene spedito a San Pietroburgo e di lì a Port-Arthur, dove decide senza volerlo, com-

(8) La questione è complessa, visto che siamo di fronte ad opere scritte a più mani, in cui il contributo di Apollinaire varia a seconda dei casi; mi limiterò dunque a riepilogare le tappe fondamentali del dibattito critico. Già M. ADEMA, Guillaume Apollinaire le mal-aimé, Paris, Plon, 1952, p. 267, riconduceva i due libri a René Delize, che di Apollinaire era stato amico d'infanzia. Per quanto riguarda La Rome des Borgia (Paris, Bibliothèque des Curieux, 1913), la paternità di Apollinaire viene negata anche oggi, benché restino fondati i dubbi in proposito di C. Debon-Tournadre, Guillaume Apollinaire de 1914 à 1918, Thèse pour le doctorat d'État, Université de Paris-Sorbonne IV, 1978. La fin de Babylone (1914) è stato invece inserito da Michel Décaudin nel primo tomo della Pléiade (G. Apollinaire, Euvres en prose, Paris, Gallimard, 1977) che fa tuttora autorità sull'argomento.

(9) Ivi, p. 1411.

(10) I due romanzi erotici furono collocati al posto che compete loro, tra le opere in prosa "maggiori” di Apollinaire, soltanto dopo la già citata edizione delle Onze mille verges (1973) e quella di ID., Les exploits d'un jeune Don Juan, préface de Michel DÉCAudin, Paris, Jean-Jacques Pauvert, 1977. Nell'introduzione al secondo, Décaudin azzardava anche una proposta di cronologia (p. 15), prescindendo dai frontespizi delle princeps, probabilmente contraffatti, e datando le due opere al periodo 1910-1913. I testi vennero poi inseriti in ID., Euvres en prose complètes, textes établis, présentés et annotés par Pierre CAIZERGuEs et Michel DeCAUDIN, Paris, Gallimard, 1977-1993, vol. III, pp. 887 1000 , ma stampati in carattere tipografico ridotto rispetto al resto del volume, quasi a nascondere il corpo del reato.

(11) Cfr. H. Werner, Les exploits d'un jeune Don Juan: un vieux problème est résolu, «Que Vlo-Ve?», IV 12, octobre-décembre 2000, pp. 110-121.

(12) Cfr. D. Delbreil, Apollinaire, cit., p. 255.

(13) «C'est alors la question de la réécriture qui intéresse la critique, l'assimilation des textes des autres par un écrivain dévoreur de livres, apte à les digérer mais surtout à les refaçonner pour en faire "sa chose" propre», ivi, p. 13. 
plici le sue disgustose pratiche erotiche, il conflitto russo-giapponese del 1905 - viene costruito come un caleidoscopio avanguardistico, in pieno accordo con gli eccessi e con le bizzarrie dell'Esprit nouveau. Il genere pornografico funge da mera impalcatura per un'opera di scoppiettante immaginazione verbale, che stempera nell'ironia (se non nella parodia) l'esaltazione "sadica" del sesso, e che trova la propria cifra nell'iperbole. Questa tendenza all'assurdo anche numerico ${ }^{14}$, resa manifesta sin dal calembour sulla leggenda di Sant'Orsola che dà il titolo al volume, viene ribadita, con intento quasi programmatico, dal cappello introduttivo commissionato a Louis Perceau, dove il libro è messo in competizione con Sade in persona ${ }^{15}$. «L'allégresse truculente, la verve picaresque, [...] l'invention délirante, la géographie sans frontières», per adoperare le parole di Michel Décaudin ${ }^{16}$, adattano al manichino del romanzo erotico un abito del tutto apollinairiano, facendo delle Onze mille verges un «Sade accommodé à la sauce rabelaisienne» ${ }^{17}$.

L'erotomania di Apollinaire non ha dunque niente di gratuito: al momento di misurarsi con il genere erotico, di cui lo attraggono le possibilità combinatorie, il poeta impiega le sue consuete risorse espressive, scombinando e riassemblando a piacere, come in un quadro cubista, le tessere della narrazione; anzi, proprio nel campo di questa letteratura "minore" egli ottiene, con le Onze mille verges, il risultato più alto della sua prima stagione di prosatore. Tuttavia, a fianco di quest'Apollinaire buongustaio della riscrittura, pornografo per gioco e per passione, ne convive un altro: alludo all'erudito di curiosa, al bibliofilo che nel 1913 dette alle stampe, assieme a Louis Perceau e Fernand Fleuret, il primo catalogo organico del cosiddetto Enfer della Nazionale di Parigi ${ }^{18}$.

L'incontro tra Apollinaire, Fleuret e Perceau risale al 1909, e ha per sfondo la saletta seminascosta dove era ospitata la collezione di opere licenziose della Richelieu: l'Enfer, per l'appunto, cui si accedeva salendo una scala a chiocciola, dopo aver ottenuto uno speciale permesso. A chi conosce lo straordinario fiuto di Apollinaire, che gli permise spesso di porsi come carrefour delle nuove arti, il suo legame con Perceau e Fleuret, con cui il poeta non condivise che una curiosità da dilettanti per la letteratura erotica, potrebbe apparire insolito. Tuttavia fra i tre, che alla Richelieu conducevano ricerche simili, nacque in breve una briosa amicizia. Così lo stesso Fleuret, vent'anni più tardi, ricordava il tenore di quel sodalizio intellettuale:

Ainsi perdions-nous tous les jours une bonne heure en bavardages, à cette table de la Réserve où nous travaillâmes côte à côte pendant plusieurs années. [...] Sa [di Apollinaire] lecture n'était jamais très assidue. Tantôt il semblait sucer distraitement des bonbons, faisant la moue, ou torturant sa bouche enfantine; tantôt, les yeux vers la coupole, il murmurait inlassablement un refrain de sa composition, toujours le même: Foutre! foutre! foutre! foutre! foutre! foutre! ${ }^{19}$

(14) La predilezione per ciò che è esagerato e caricaturale risalta, per esempio, dal gusto per la moltiplicazione, che ingigantisce in modo surreale le pratiche sessuali dei protagonisti. «Il ne quitta le con de Culculine qu'après y avoir déchargé trois fois, tandis qu'elle-même déchargeait dix fois», $G$. Apollinaire, Les onze mille verges, cit., p. 48.

(15) «"Plus fort que le marquis de Sade", c'est ainsi qu'un critique célèbre a jugé Les Onze Mille Verges, le nouveau roman dont on parle à voix basse dans les salons les plus cossus de Paris et de l'étranger. Ce volume a plu par sa nouveauté, par sa fantaisie impayable, par son audace à peine croyable. Il laisse loin derrière lui les ouvrages les plus effrayants du divin marquis», cfr. ivi, p. 16.

(16) ID., Euvres en prose, cit., vol. III, p. 1326.
(17) ID., Les onze mille verges, cit., p. 19.

(18) L'Enfer de la Bibliothèque Nationale. Iconobio-bibliographie descriptive, critique et raisonnée, complète à ce jour de tous les ouvrages composant cette célèbre collection, avec un index alphabétique des titres et noms d'auteurs, par G. ApOLlinaIRE, F. Fleuret, L. Perceau, Paris, Mercure de France, 1913.

(19) F. Fleuret, De Gilles de Rais à Guillaume Apollinaire, Paris, Mercure de France, 1933, p. 285. A sua volta, nell'introduzione de L'Euvre libertine des poètes du XIX siècle, Apollinaire designa Fleuret con lo pseudonimo «Le petit neveu du Baffo»: cfr. L. CAMPA, Sous le signe d'Aphrodite et d'Athéna: Guillaume Apollinaire et l'cuvre libertine des poètes du XIX siècle, «Littératures classiques», XXXI 1997, p. 205. 
Sia detto en passant che, quanto a bizzarrie, Fleuret e Perceau non erano da meno del loro più celebre compagno di avventura. Poeta e mistificatore culturale, il primo costeggiò molti dei protagonisti della Parigi d'inizio secolo, tra cui Max Jacob e Jean Cocteau, ma restò ancorato ad una lirica stralunata e nostalgica, fatta di dotte evocazioni dell'Ancien Régime; il secondo incarnava il tipo stesso del poligrafo ${ }^{20}$, e come tale consacrò la propria inesauribile attività a edizioni e a bibliografie di testi erotici, ma non smise per questo i panni del giornalista né quelli, ancora più atipici per la temperie dell'epoca, dell'ex-blanquiste, militante socialista e diffamatore dell'armée, che gli costarono il carcere.

Già prima che il repertorio bibliografico dedicato all'Enfer venisse dato alle stampe, fruttando ai suoi autori una certa popolarità tra gli appassionati di curiosa, Apollinaire aveva moltiplicato i fronti del suo lavoro erudito. Nel 1908, infatti, si era impegnato in veste di curatore di "classici erotici" presso la Bibliothèque des Curieux, una piccola casa editrice che aveva sede in piazza Furstenberg, a Parigi. I proprietari, i fratelli Georges e Robert Briffaut, avevano appena congedato un altro progetto editoriale per dedicarsi alla letteratura erotica, sperando di trovarvi un campo più redditizio, e non ricalcavano certo il profilo dei mecenati viziosi. Le loro collane, estranee a mire apertamente clandestine, si inserivano nella voga divulgativa tipica della Belle Époque: i testi erano scelti, e si limitavano ai nomi più noti della tradizione del genere; i passaggi osceni erano censurati, così che anche le pubblicazioni più audaci restavano ben al di qua del confine tra erotismo piccante e pornografia. Apollinaire stese le prefazioni di cinque dei sei volumi con cui esordì la prima collana dei Briffaut, «Les Maîtres de l'Amour», che comprendeva opere di Aretino (2 voll.), Sade, Mirabeau, Nerciat (3 voll.), Baffo e Chorier; s'occuperà, in seguito, della metà dei titoli stampati nelle quattro serie seguenti ${ }^{21}$.

Quale ruolo avesse per Apollinaire questa attività critica, da molti rimproveratagli come semplice occasione di guadagno - è noto che il poeta, in quel giro d'anni, non era alieno da preoccupazioni economiche - emerge dall'intenzione, manifestata nel 1914 e poi abbandonata durante la guerra ${ }^{22}$, di riunire i suoi interventi in un volume che avrebbe dovuto intitolarsi Les diables amoureux. Tale progetto non rappresentava un capriccio stravagante; si collocava, al contrario, nel solco di una precisa tradizione editoriale, ben radicata nella seconda metà del XIX secolo, che aveva avuto la sua consacrazione nei Curiosa di Alcide Bonneau (1836-1904) ${ }^{23}$ : un libro pensato

(20) Come riassume il titolo di V. LaBaume, Louis Perceau. Le polygraphe (1883-1942), Paris, Jean-Pierre Faur éditeur, 2005. A conferma di ciò, l'approccio di Perceau alla letteratura erotica si rivela diverso da quello dei "poeti" Apollinaire e Fleuret: la sua attività di critico mira alla rivalutazione dell'intero genere letterario, non alla brillante rivisitazione della perla nascosta o del frammento erudito. Ne danno testimonianza, ad esempio, le notizie per le edizioni clandestine di Maurice Duflou, gli studi su Brantôme e Jarry, o ancora l'antologia del 1935 Théophile de Viau et les Libertins. Cfr. ivi, pp. 142, 225-240.

(21) Apollinaire firmò numerose notizie per «Le Coffret du Bibliophile», una collana che riuniva classici minori e di formato più ridotto. Nel progetto dei Diables amoureux molte di queste introduzioni, solitamente assai brevi e di dubbia originalità, andranno ad affiancare i saggi "maggiori" scritti per «Les Maîtres de l'Amour» e alcune delle schede curate da Apollinaire per l'Enfer.

(22) Michel Décaudin ha ricostruito la storia del testo in G. ApollinAIRE, Les diables amoureux, Paris, Gallimard, 1964 , pp. 7-18, e nelle note a ID., Euvres en prose, cit., vol. III, pp. 1277-1284. Il manoscritto dei Diables amoureux fu depositato nel 1914 al «Mercure de France», dove rimase sino alla morte di Apollinaire; fu quindi ceduto alle edizioni della «Nouvelle Revue Française» nel luglio del 1919. All'altezza del 1925, probabilmente in vista di un'edizione parziale, il codice fu smembrato e alcune delle sue parti smarrite. Décaudin si è dunque basato, per l'edizione critica, su un autografo incompleto, integrandone la lezione con i contributi usciti per la «Bibliothèque des curieux». Un'accurata descrizione del dossier originale, fatto di pagine incollate, anche a stampa, e postille di mano di Apollinaire e del suo factotum Jean Mollet, è in Eros invaincu: la bibliothèque Gérard Nordmann, Genève, Éditions Cercle d'Art, 2004, p. 230.

(23) A. Bonneau, Curiosa. Essais critiques de littérature ancienne ignorée ou mal connue, Paris, Isidore Liseux, 1887. 
come «une sorte de supplément à l'histoire de la littérature italienne et de la littérature française ${ }^{24}$, formidabile per dottrina e per intuito, in cui venivano raccolti i saggi introduttivi scritti dall'autore per le edizioni clandestine di Isidore Liseux (18351894). Erudito di professione, Bonneau aveva collaborato al Grand dictionnaire di Pierre Larousse e godeva di una solida fama di bibliofilo; non è perciò sorprendente che Apollinaire, che ne ammirava «l'érudition élégante et inépuisable» ${ }^{25}$, avesse fatto dei Curiosa un livre de chevet, e mirasse a realizzare un piano di lavoro simile nonostante le limitazioni impostegli dai Briffaut (rimozione dei materiali lessicali più scomodi, alleggerimento dell'apparato critico). Con ogni probabilità, nelle intenzioni di Apollinaire, una ricognizione della letteratura erotica di tale portata avrebbe dovuto garantirgli una reputazione "ufficiale" di specialista, affine a quella di Bonneau e spendibile con altrettanto profitto nel vulcanico mercato librario della Parigi d'avant guerre. La piena legittimazione del proprio ruolo di savant, apparentemente estranea all'Apollinaire poeta, resterà a lungo una delle prime ambizioni del curatore dell'Enfer e della Bibliothèque des Curieux.

Se da quanto detto emerge che i Diables amoureux nacquero per emulazione dei Curiosa di Bonneau, altra cosa è chiedersi quale fosse l'autentico significato dell'operazione apollinairiana, o ancora, ribaltando i termini della questione, in che modo e in che dosi la personalità dell'Apollinaire "maggiore" si specchi nelle prefazioni in margine ai volumi della Bibliothèque des Curieux. A mio avviso, all'interno del quadro più generale qui tracciato, gli scritti sul poeta veneziano Giorgio Baffo $(1694-1768)^{26}$ - il maggior lirico in dialetto, assieme a Domenico Tempio, del nostro Settecento, autore di culto del panorama libertino e del dorato tramonto della Serenissima - si prestano ad un'interpretazione complessiva dei rapporti tra il poeta e la letteratura di second rayon. Infatti, benché non spicchino né per numero né per profondità critica tra quelle dedicate a Sade o ad Aretino, le pagine su Baffo rappresentano, per le categorie chiamate in gioco, quasi una mise en relief dell'approccio di Apollinaire alla materia erotica.

Chi sbuca, a Venezia, in campo San Maurizio, e si trova di fronte lo splendido palazzo Bellavite, dove Baffo passò i suoi ultimi anni (1759-1768), non può fare a meno di notare la lapide consacrata alla sua memoria: «Qui visse Giorgio Baffo, poeta dell'amore, che ha cantato con la massima libertà e con grandiosità di linguaggio. Guillaume Apollinaire, 1910». La targa è così recente (1987) da far sorgere il dubbio che si tratti più che altro di una patente di legittimità per il grande erotico veneziano, una scorciatoia verso i piani alti della poesia. Tuttavia nel 1910 Apollinaire - che forse, complici le sue passeggiate tra i bouquinistes parigini ${ }^{27}$, già conosceva l'opera

(24) Ivi, p. VII.

(25) G. Apollinaire, Euvres en prose, cit., vol. III, p. 658.

(26) Di nobili origini, ma di collocazione modesta in seno al patriziato veneto, Giorgio Baffo percorse senza infamia e senza lode il cursus honorum della Serenissima. Fu eletto alla Quarantia, ma non occupò mai incarichi di particolare prestigio; visse una vita tutto sommato irreprensibile, ma non priva di suggestioni culturali: si scagliò a più riprese, per esempio, contro Carlo Goldoni e Pietro Chiari, recitando da protagonista nella nota querelle teatrale del tempo. Ai suoi componimenti licenziosi e satirici, che gli valsero ben presto la fama di epicureo, assicurò vasta diffusione manoscritta; ne rifiutò sempre, tuttavia, l'edizione a stampa, che uscì postuma, sine loco (ma in realtà a Londra), nel
1771. Su Baffo, al di là delle numerose (ma spesso scorrettissime) edizioni parziali, si vedano almeno $\mathrm{G}$. BAFFO, Raccolta universale delle opere, Milano, Longanesi, 1971, e all'interno di questo volume l'introduzione di E. Bartolini, Giorgio Baffo o di tre figure, pp. 9-57; cfr. inoltre l'ampio saggio, ancora valido quanto a prospettive critiche, di $\mathrm{P}$. Del Negro, La "poesia barona" di Giorgio Baffo "Quarantiotto": politica e cultura nella Venezia di metà Settecento, «Comunità»184, XXXVI, 1982, pp. 312-425, e a cura dello stesso G. BAFFo, Poesie, Milano, Mondadori, 1991.

(27) Tra i libri appartenuti ad Apollinaire, e ora conservati alla Bibliothèque historique de la Ville de Paris, è presente un esemplare delle Opere di Baffo (RACCOLTA COMPLETA / DELLE OPERE / DI / GIORGIO BAFFO / VENETO // CO- 
di Baffo - intraprese realmente delle ricerche sul poeta libertino, curando il volume della Bibliothèque des Curieux a lui dedicato e stendendo per l'occasione un celebre saggio introduttivo ${ }^{28}$.

La "scoperta" di Baffo da parte di Apollinaire può essere ricondotta alle letture preparatorie per l'Enfer, di cui la princeps baffiana del 1771 costituiva, d'altronde, uno dei piatti forti. Ancora nella préface alla ristampa del catalogo, stesa da Fleuret e Perceau nel 1919, «les Poésies de Baffo, dont la licence frappe la Lyre d'un plectre éperdu $\gg^{29}$, venivano additate tra i testi più significativi dell'Enfer, capaci di legittimare in senso divulgativo, per il loro rilievo storico ed artistico, l'intera operazione bibliografica. Nel fondo perduto della Nazionale, Apollinaire ebbe modo di visionare l'edizione londinese del 1771 (la prima silloge di Baffo uscita a stampa, come si è detto) ${ }^{30}$ e soprattutto quella parigina del 1884, a cura di Alcide Bonneau e Isidore Liseux ${ }^{31}$. Si tratta di un'edizione estremamente lussuosa, ornata dallo stesso ritratto presente nella Raccolta universale delle opere (Cosmopoli, 1789) e composta da quattro volumi di ampio formato, che raccolgono un corpus di 755 componimenti. La traduzione, stampata con il testo veneziano a fronte, viene annunciata come letterale per la prima volta: in effetti l'edizione delle Euvres complètes di Baffo, curata otto anni prima da Antoine Ribeaucourt ${ }^{32}$, non solo proponeva un linguaggio meno preciso di quello di Bonneau, ma rinunciava alla resa in versi, proponendo brevi prose divise in couplets $^{33}$.

L'eccellente livello tipografico e critico dell'edizione del 1884 era dovuto soprattutto allo zelo di Isidore Liseux, uno dei più notevoli editori della fine del secolo, allievo e continuatore dell'opera di Poulet-Malassis, cui è stato spesso paragonato ${ }^{34}$. Dal 1875 al 1894 uscirono per le sue cure un centinaio di titoli, per lo più testi latini o italiani del Rinascimento, ma anche un gran numero di pubblicazioni licenziose, distribuite sul triplice versante della saggistica contemporanea, delle traduzioni in inglese e dei classici dell'erotismo. Le edizioni di Liseux, sempre destinate a tirature

STANTINOPOLI 1860, 2 voll. di pp. 307 e 300). Si tratta di una tipica edizione "clandestina", di piccolo formato $\left(12^{\circ}\right)$ e di scarso pregio tipografico (manca anche il ritratto dell'autore), che riproduce l'impostazione della stampa di Cosmopoli del 1789. Probabilmente Apollinaire ne entrò in possesso durante le sue promenades tra le bancarelle dei librai, che egli descrive nell'Essai sur la littérature sotadique au XIX siècle (G. ApOLLINAIRE, Euvres en prose, cit., vol. III, pp. 855-864). Il libro è privo di postille autografe, e il secondo tomo si presenta addirittura intonso, cioè con le pagine non rifilate; ma Apollinaire ebbe cura di citarlo nella bibliografia acclusa all'edizione del 1910 (p. 16) a fianco delle stampe di Londra, Cosmopoli e Parigi.

(28) Questa prefazione sarà ristampata più volte a margine delle edizioni semiclandestine di Baffo, anche moderne: cfr. ad esempio G. BAfFo, Poesie, Milano, Sunday Press italiana, 1972, e ID., Poesie, La Spezia, Club del libro Fratelli Melita, 1985.

(29) L'Enfer de la Bibliothèque Nationale. Bibliographie méthodique et critique de tous les ouvrages composant cette célèbre collection, avec une préface, un index des titres et une table des auteurs, par G. Apollinaire, F. Fleuret, L. Perceau, Bibliothèque des curieux, Paris, 1919.

(30) L'Enfer ne conservava due copie: cfr. ivi, pp. $118,146$.

(31) POÉSIES COMPLÈTES / DE / GIOR-
GIO BAFFO / En dialecte Vénitien / littéralement traduites pour la première fois, avec le texte en regard / ORNÉ DU PORTRAIT DE L'AUTEUR // TOME PREMIER // Imprimé a cent exemplaires / POUR ISIDORE LISEUX ET SES AMIS / PARIS 1884, 4 voll. di pp. 362, 365, 372 e 386.

(32) CEUVRES COMPLETES / DE / G. BAFFO, / TRADUITES DU VÉNITIEN / PAR / $A$. RIBEAUCOURT // PREMIER VOLUME // PARIS - 1876, pp. 175 . E un'edizione a bassissima tiratura (20 esemplari) che raduna soltanto 154 componimenti. Nella Note préliminaire, Baffo viene dipinto come «celui qui a mérité la palme de l'obscénité» tra tutti gli scrittori licenziosi d'Italia (p. 1).

(33) L'effetto ricorda il poème en prose, che del resto prese piede, come è noto, proprio dalle traduzioni di poesia: cfr. N. VINCENT-MUNNIA, Les premiers poèmes en prose: généalogie d'un genre, Paris, Champion, 1996.

(34) «À consulter la liste de ses éditions, on peut considérer Liseux comme le successeur de PouletMalassis, le génie d'avoir publié Les Fleurs du mal en moins», L'Enfer de la bibliothèque. Éros au secret, sous la direction de M.F. QUIGNARD et R.J. SECKEL, Paris, Bibliothèque nationale de France, 2007, p. 212. Sulla figura di Liseux si veda anche P. ADAMY, Isidore Liseux, 1835-1894: un grand «petit éditeur», Bassac, Plein Chant, 2009. 
d'élite, venivano confezionate con prudenza e senso pratico: Les Kama Sutra de Vatsyayana, prima traduzione francese dell'opera sanscrita, fu pubblicato con una copertina castigata e con l'avviso che, trattandosi di un'edizione privata, ne erano proibite la vendita e l'esposizione nei luoghi pubblici"is . Ciò non toglie che si trattasse di volumi curati sin nei minimi dettagli, spesso in più tomi, ricchi di incisioni e raffinati nella scelta di carta e inchiostri; tanto che il Liseux degli ultimi anni, più che dalla censura, dovette guardarsi dalla bancarotta. Da parte sua, Bonneau secondò fedelmente il progetto dell'editore, mettendogli a disposizione le sue competenze erudite. Le Poésies complètes di Baffo testimoniano anch'esse la felicità di questo connubio.

Che Apollinaire conoscesse l'edizione Bonneau-Liseux ci viene assicurato, come si è detto, dalla recensione del libro nell'Enfer. Un confronto più serrato tra la traduzione del 1884 e quella che Apollinaire approntò nel 1910 per «Les Maîtres de l'Amour ${ }^{36}$ consente di spingersi oltre. La versione di Bonneau non presenta censure, e si vuole anzi letterale come enunciato dal frontespizio; semmai, rispetto all'espressionismo vernacolare del testo di partenza, il traduttore opera qualche ovvia e necessaria smorzatura (mona: moniche; sborar: décharger; chiavar: enfiler). Viceversa Apollinaire, che deve fare i conti con le ambizioni "commerciali" dei Briffaut, da una parte elimina il testo a fronte, dall'altra smussa gli angoli più spigolosi della traduzione francese di Bonneau (branler: manuéliser; cas: vit). Ciò non gli impedisce di saccheggiarne il lessico e di ricalcarne talvolta intere strofe, così che, malgrado la promessa di una «traduction nouvelle», la sua versione si configura come un plagio a bella posta, per giunta più scorretto dell'originale. Ne trae conferma una considerazione d'ordine più generale: il fascino dell'Apollinaire traduttore dall'italiano, cui pure si è dimostrata sensibile certa critica non superficiale ${ }^{37}$, ha ben pochi fondamenti, basandosi su un falso mito abilmente coltivato dal poeta stesso.

Appare legittimo, a questo punto, domandarsi se l'edizione curata da Apollinaire - fatti salvi i suoi intenti divulgativi, certamente meritori - abbia qualche altro motivo d'interesse. Quanto al libro in sé, credo che la risposta sia negativa: il Baffo di Apollinaire non aggiunge nulla a quello di Bonneau, diffondendone una copia edulcorata presso un pubblico più ampio ${ }^{38}$. Resta tuttavia incerto se questa prospettiva, che è poi quella del lettore moderno, fosse la stessa di Apollinaire; in altre parole, a dispetto degli esiti realmente modesti dell'iniziativa editoriale, va indagato il valore che quest'ultima ebbe per l'editore, e se il suo incontro con Baffo nasconda altro da un lavoro di routine, eseguito per ragioni meramente economiche ${ }^{39}$. A tal fine, prima di leggere la prefazione del 1910, riuscirà utile l'analisi di alcuni testi che, sebbene rimasti a margine del volume, interagiscono con esso e ne condividono la chiave interpretativa.

(35) Cfr. Éros au secret, cit., p. 213. Si veda anche L'Enfer de la Bibliothèque Nationale, cit., p. 75.

(36) L'CEUVRE / du Patricien de Venise / Giorgio Baffo // Sonnets - Madrigaux - Canzoni / Capitoli / Traduction nouvelle // Introduction, essai bibliographique et notes / par / GUILLAUME APOLLINAIRE // Ouvrage orné d'un portrait hors texte // PARIS / BIBLIOTHEQQUE DES CURIEUX / MCMX, pp. 288.

(37) Alludo al saggio di A. Fongaro, Apollinaire traducteur des "Sonnets luxurieux" de l'Aretin, «Que Vlo-ve?» IV 5, janvier-mars 1999, pp. 1-16, che indaga la ricezione del testo di Aretino sull'asse Bonneau-Apollinaire, evidenziando le ingiustificabili storpiature presenti nella traduzione del poeta. L'analisi di Fongaro è rigorosa, ma al di là di confutare una volta di più il presunto bilinguismo di Apollinaire, che in italiano dimostra competenze assai limitate, ribadisce soltanto l'assoluta trascuratezza (verrebbe da dire: lo scarso interesse) con cui il poeta traduceva questi testi.

(38) Il giudizio non vale solo nel caso di Baffo, ma può essere esteso, con qualche rara eccezione, all'intera attività svolta da Apollinaire per la Bibliothèque des Curieux. Lo sosteneva su «Carrefour», a pochi mesi dall'edizione di Décaudin dei Diables amoureux (1964), P. PIA, À l'entour d'Apollinaire, ora in «Que Vlo-ve?» I 24, avril 1980, pp. 19-24.

(39) Spesso la collaborazione di Apollinaire con i Briffaut è stata liquidata in tal modo: cfr. ivi, pp. 1920. 
Durante il censimento della biblioteca di Apollinaire sono state ritrovate alcune brevi notizie scritte per il catalogo della Bibliothèque des Curieux. Queste prières d'insérer, rimaste inedite sino al 1987, dovrebbero essere di mano del poeta stesso ${ }^{40}$, che vi traccia un riassunto della propria operazione critica. In particolare, nel cappello pubblicitario a L'Euvre du Patricien de Venise Giorgio Baffo si legge:

Giorgio Baffo, tuteur de l'aventurier Casanova, a écrit en dialecte vénitien l'épopée de l'amour. C'est une œuvre exquise qui évoque la Venise galante du XviII ${ }^{\mathrm{e}}$ siècle, le Carnaval, les Nonnes, les Gondoliers, l'Osteria, les Casinos.

Baffo fut un véritable et grand poète. Il n'a chanté, il est vrai, que le plaisir sensuel, mais avec une verve, une souplesse d'imagination, un souffle incroyables. Baffo a chanté surtout la Vénus prodigieuse chère aux Italiens, et il l'a chantée comme la chose la plus naturelle du monde; mais ses aventureux exploits, ses grasses bonnes fortunes, ses rêves de débauches surhumaines sont présentés avec un esprit endiablé, une bonne humeur communicative, une drôlerie des plus comiques, voire même une grâce qu'on s'attendrait peu à trouver en de pareils sujets.

Baffo est sans contredit le poète le plus expressif du XVIII ${ }^{e}$ siècle italien. Il a d'ailleurs écrit aussi quelques contes qui ne manquent pas de charme et d'originalité; mais ce sont bien ses Canzoni, ses sonnets, qui assureront la pérennité de son nom. ${ }^{41}$

In questo caso la dipendenza di Apollinaire dall'Avertissement che apre l'edizione Bonneau-Liseux si fa addirittura imbarazzante: frasi intere ${ }^{42}$, tolte di peso dall'introduzione del 1884, vengono ritoccate e incollate alla meglio in una sorta di pastiche pubblicitario. La réclame, però, si chiude con un giudizio di valore altisonante («sans contredit»), che se da un lato assolve ai doveri della promozione sembra, dall'altro, lasciar filtrare la vera opinione di Apollinaire. L'elogio trova infatti un'eco significativa nell'Enfer, dove Apollinaire, catalogando l'edizione Bonneau-Liseux, fa seguire ad una dettagliata scheda bibliografica due righe apparentemente avulse dal contesto: «On peut regarder Baffo comme un grand poète lyrique, comme un des plus grands poètes du XVIII ${ }^{\mathrm{e}}$ siècle. Il écrivait en dialecte vénitien ${ }^{43}$. Per comprendere la portata di quest'affermazione, e in particolar modo dell'aggettivo lyrique, occorre fare un passo indietro e considerare il modo in cui Apollinaire si rapportò alla vulgata corrente sulla figura di Baffo.

Baffo godette, già tra i suoi contemporanei, della scomoda fama di poeta osceno; una fama confortata, sotto il profilo editoriale, dalla stampa londinese del 1771, che anteponeva le prove erotiche, di più sicuro successo, a quelle filosofiche e politiche. Posti davanti alla condanna degli ambienti "ufficiali" della letteratura, i difensori di Baffo avevano fatto ricorso alla famosa massima di Marziale: «lasciva nobis est pagina, vita proba» ${ }^{44}$. Pierre-Louis Ginguené, in una scheda biografica pubblicata a Parigi nel

(40) Come ipotizzato da Pierre Caizergues nella chiosa al Catalogue de la bibliothèque de Guillaume Apollinaire, établi par Gilbert BOUDAR, Éditions du Centre National de la Recherche Scientifique, $\mathrm{Pa}$ ris, 1987, vol. II, p. 11.

(41) Ivi, p. 91.

(42) «Baffo [...] fut un grand poète et surtout un poète unique. Il n'a chanté que le plaisir sensuel, mais avec quelle originalité d'esprit, quelle incroyable fécondité d'imagination! [...] Baffo chante la Venus prodigieuse, chère aux Italiens, comme si c'était la chose la plus naturelle du monde; il raconte ses exploits, ses bonnes fortunes, ses jouissances solitaires ou partagées, et décrit des débauches ima- ginaires ou réelles [...]. Toutes ses folies se pardonnent à la faveur de l'esprit, de la bonne humeur, de la drôlerie vraiment comiques, et parfois même de la grâce que l'auteur répand à pleines mains», $A$. Bonneau, Avertissement, in G. BAFfo, Poésies complètes, cit., pp. X-XIII.

(43) L'Enfer de la Bibliothèque Nationale, cit., p. 72 .

(44) L'aforisma, citato assai di frequente in simili casi, è in MarzIALE, Epigrammata, I, IV, 8. Quest'interpretazione moralizzante della figura di Baffo fu inaugurata da G. MoschINI, Della letteratura veneziana del secolo XVIII fino a' nostri giorni, 4 voll., Venezia, 1806-08, vol. II, pp. 152-153. 
1811, arrivava a fare di Baffo un personaggio ai limiti della schizofrenia, sostenendo che il veneziano «parlait comme une vierge et écrivait comme un satyre» ${ }^{45}$. Di questa voce infondata, ma utile a scansare una damnatio memoriae sempre in agguato, dà conto anche Apollinaire ${ }^{46}$, che però si limita a riportare la notizia senza darle troppa importanza. Simili scrupoli in fatto di morale si ponevano, come è ovvio, al di fuori degli interessi del poeta, che del genere erotico apprezzava viceversa la liberté, intesa nel senso più apollinairiano del termine ${ }^{47}$ : una libertà etica ed estetica ad un tempo, di cui la letteratura di second rayon, avendo origine proprio dall'infrazione delle regole vigenti, costituiva una perfetta incarnazione ${ }^{48}$.

Tuttavia, pur non cadendo in questo trabocchetto apologetico, Apollinaire non percorreva neppure la strada della rivalutazione filosofica del poeta, battuta invece dalla critica più recente. La prefazione del 1910 non mostra alcuna curiosità per il Baffo epicureo, né per i suoi rapporti (a dire il vero abbastanza evanescenti) con l'illuminismo "radicale" di Holbach ed Helvétius; l'autentico significato del termine libertin, che tra Sei e Settecento procede sempre sul doppio binario della morale e dell'ideologia, designando tanto il depravato quanto l'esprit fort, sembra ignoto ad Apollinaire. A suscitare il suo interesse è piuttosto il valore esemplare, di testimonianza storica delle poesie di Baffo. La tesi era tutt'altro che innovativa ${ }^{49}$, ma nella prosa di Apollinaire si colorava di un fascino inedito:

Le Baffo était content de son époque, il était heureux de vivre, et de vivre à Venise, ville amphibie, cité humide, sexe femelle de l'Europe.

Sans le Baffo, on n'imaginerait pas tout ce que fut la décadence pleine de volupté de la Sérénissime République. Par lui nous connaissons la vie sexuelle de Venise, les fêtes, les Osterie, les Casinos, le Jeu, les Ballerines, les Nonnes libertines ${ }^{50}$.

D'altronde, benché le immagini siano così vivide da risultare tipicamente apollinairiane, anche nel ritratto di questa Venezia crepuscolare Apollinaire segue le orme di Bonneau ${ }^{51}$, e così fa nel resto del saggio, quando tratta dell'antenata di Baffo che, dopo essere stata rapita dai turchi, sarebbe diventata una sultana ottomana, oppure riporta alcuni episodi dei Mémoires di Casanova ${ }^{52}$, o ancora trascrive riga per riga informazioni dubbie o persino inesatte ${ }^{53}$. Quest'imitazione senza troppe pretese, che occupa i tre quarti della prefazione, avrebbe dovuto garantire ad Apollinaire il palcoscenico su cui esibire la propria erudizione in fatto di curiosa.

All'interno di questo schema, di cui si sono chiariti in precedenza i presupposti, il poeta sa ritagliarsi però dei margini di autonomia. Nel caso del saggio su Baffo, le digressioni in cui fa capolino il vero Apollinaire, finalmente "libero" dalle proprie

(45) Cfr. P. Del Negro, La "poesia barona", cit., p. 319.

(46) Cfr. G. Apollinaire, Les diables amoureux, cit., p. 90.

(47) Vengono in mente alcuni passi del già citato Essai sur la littérature sotadique au XIX siècle, dove Apollinaire sostiene, quanto al soggetto del proprio intervento: «je l'ai choisi parce qu'il est libre, sans jeu de mots» (ID., Euvres en prose, cit., vol. III, p. 855), per poi ribadire piccato le limitazioni con cui tale repertorio è messo a disposizione del pubblico.

(48) Cfr. L. CAMPA, Sous le signe d'Aphrodite, cit., p. 210.

(49) Cfr. G. Ferrari, De la littérature populaire en Italie, «Revue des Deux Mondes», XVIII 1839, pp. 697-698. Si tratta dell'altro grande "serbatoio", assieme agli studi di Bonneau, da cui attinge Apollinaire per la propria edizione.

(50) G. Apollinaire, Les diables amoureux, cit., p. 95.

(51) Cfr. A. Bonneau, Avertissement, cit., p. XIII.

(52) Cfr. ivi, pp. VIII-X. Apollinaire si limita, per così dire, ad allungare il brodo, citando per intero i sonetti di Baffo dedicati all'illustre ava e i brani di Casanova che riguardano il poeta. Bonneau, con maggiore eleganza, vi alludeva soltanto.

(53) Mi riferisco rispettivamente alla supposta povertà di Baffo e al suo impenitente rifiuto di prendere moglie (cfr. Diables amoureux, cit., p. 89; Avertissement, cit., p. XVII). 
ambizioni di savant, si distribuiscono all'inizio e alla fine del testo, quasi ad abbracciare con una costruzione ad anello il bagaglio di notizie bio-bibliografiche. L'esordio, ad esempio, presenta un ritratto non convenzionale di Baffo:

Baffo, ce fameux vérolé, surnommé l'obscène, que l'on peut regarder comme le plus grand poète priapique qui ait jamais existé et en même temps comme l'un des poètes les plus lyriques du XVIII' siècle, écrivait dans ce patois vénitien qu'ont illustré un grand nombre d'ouvrages remarquables dans tous les genres. [...]

Le patois vénitien a une douceur unique. La grâce et la mollesse s'y mêlent dans des proportions si justes qu'il favorise avant tout le lyrisme érotique bien qu'une littérature patoise soit presque toujours satirique. ${ }^{54}$

Come è facile notare, qui l'erudizione passa in secondo piano. Al di là dei dettagli piuttosto fantasiosi ${ }^{55}$ con cui viene introdotto il personaggio, Apollinaire torna ad insistere sulla grandezza di Baffo in quanto poète, non limitandosi all'elogio di Casanova $^{56}$, ma elevando lo statuto del libertino veneziano da un tecnico priapique ad un più nobile, e intimamente apollinairiano, lyrique. Il dubbio che, dietro la ripetizione dell'aggettivo attribuito a Baffo nell'Enfer, ci sia qualcosa di più di una coincidenza, diventa certezza qualche riga più sotto, dove Apollinaire parla espressamente di «lyrisme érotique». Ora, che la letteratura erotica seducesse Apollinaire anche perché poneva il problema della lingua e dello stile, è già stato acutamente individuato dalla critica $^{57}$. Tuttavia la parentesi sui dialetti italiani che apre questa introduzione scende più in profondità: il ruolo del dialetto in Italia, scrive Apollinaire, è stato importante sin dai tempi di Dante ${ }^{58}$, ma generalmente ha riguardato la letteratura satirica. La poesia veneziana di Baffo, invece, pur appartenendo ad un campo "minore" come quello erotico, ha tutte le prerogative del genere più alto, la lirica appunto, e assurge quindi alla poesia tout court.

Mi si permetta un'ultima citazione, tratta questa volta dalla conclusione del saggio:

Ce poète qui fit souvent songer à Horace avait avant tout du bon sens, et la raison ne gênait point son lyrisme. [...]

Les poésies manuscrites du Baffo couraient la ville. Les jeunes femmes les lisaient en goûtant des sorbets. Cette société raffinée qui vivait à l'anglaise était frappée par un lyrisme auquel les poètes de l'époque ne l'avaient point accoutumée. [...]

Le Baffo viole la poésie, c'est entendu. Toutefois, cet événement a la grandeur et la valeur symbolique d'une fête vénitienne. Chaque année, le Doge épousait la mer. ${ }^{59}$

In queste ultime righe il termine lyrisme si fa addirittura martellante, preparando il terreno alla sconvolgente rivelazione della chiusa: proprio Baffo, il violeur della poesia, è in realtà una delle reincarnazioni di Orfeo ${ }^{60}$. Infatti l'allusione finale alla Festa

(54) G. Apollinaire, Les diables amoureux, cit., p. 85 .

(55) L'affermazione di Apollinaire per cui Baffo, da bravo libertino, sarebbe stato malato di sifilide (vérolé), si fonda sul presunto valore autobiografico di sonetti come Puttana buzarona! Più no posso, la cui attribuzione a Baffo è peraltro dubbia: cfr. P. Del Negro, La "poesia barona", cit., p. 325.

(56) «M. Baffo, sublime génie, poète dans le plus lubrique des genres, mais grand et unique». Cfr. G. Apollinaire, Les diables amoureux, cit., p. 90.

(57) Si veda ancora una volta il bel saggio di L.
CAmpa, Sous le signe d'Aphrodite, cit., p. 214, che studiando L'Euvre libertine des poètes du XIX siècle, un'altra delle edizioni curate da Apollinaire per la Bibliothèque des Curieux, giunge a conclusioni in gran parte analoghe.

(58) Cfr. G. Apollinaire, Les diables amoureux, cit., p. 85 .

(59) Ivi, pp. 95-96.

(60) Difficile non ricordare, a questo proposito, le pagine tuttora illuminanti di PH. RENAUD, Lecture d'Apollinaire, Lausanne, L'âge d'homme, 1969, p. 180: «Le lyrisme se présenterait, ainsi, comme 
della Sensa, che celebrava lo sposalizio tra Venezia e il mare ${ }^{61}$, conferisce all'erotismo del poeta libertino una connotazione quasi sacra, facendone un simbolo del ciclo di morte e rinascita della natura. Estraneo ad una poesia schiava della mimesis aristotelica, Baffo entra a far parte del ristretto novero dei poetae theologi, i poeti, cioè, che hanno ereditato le stimmate dello spirito orfico ${ }^{62}$. Non va dimenticato che nelle Onze mille verges, dopo aver recitato il sonetto erotico che ho ricordato in apertura ${ }^{63}$, il principe Vibescu veniva proclamato - con un'ironia che il lettore di Apollinaire sa di dover prendere sul serio - «poète archi-divin».

Azzarderei il passaggio successivo. Apollinaire, in Baffo, cerca soprattutto se stesso. Poco importa che il suo italiano sia zoppicante, o che le esigenze editoriali lo costringano a sopprimere il testo originale: nella douceur, nella grâce, nella mollesse di quei versi in veneziano Apollinaire ritrova, a suo modo, la musicalità orfica. È bene ribadire che, all'altezza del 1910, prima di Zone e della "discesa all'inferno" rappresentata dalla guerra, Apollinaire è ancora il poeta del «triste et silencieux délire» ${ }^{64} \mathrm{di}$ sapore simbolista, l'ammiratore di Verlaine che, nel Cortège d'Orphée, si vanta del proprio potere creativo associato alla musica: «Mes doigts sûrs font sonner la lyre» ${ }^{65}$. Va da sé che Baffo, a differenza di Apollinaire, non dovrà confrontarsi con il caos della modernità; rimarrà un Orfeo dei tempi antichi, ormai irraggiungibile nella purezza del suo «lyrisme érotique».

La collaborazione tra Apollinaire e i fratelli Briffaut appare fondata in modo inequivocabile sulle esigenze del mercato prima che su quelle della poesia. Il compenso che il poeta ottiene per le sue curatele non è tanto alto da consentirgli una riflessione lungimirante su ogni autore: non sorprende che, per sbrigare il proprio compito, egli s'affidi ad un copia e incolla dalle benemerite edizioni Bonneau-Liseux, soddisfacendo in pieno, tra l'altro, l'idea di alta divulgazione professata dagli editori. Resta tuttavia, nell'Apollinaire dei Diables amoureux, qualcosa di non riconducibile alla pratica erudita. Nonostante la volontà di oggettivare il proprio campo di ricerca per competere con gli specialisti del settore, lo sguardo rimane quello del poeta vate

un art magique où le poète [...] se donne pour un initié qui prend des risques pour accéder à de plus grands pouvoirs».

(61) La cerimonia aveva luogo in occasione dell'Ascensione di Cristo (Sensa in dialetto veneziano) e simboleggiava il dominio marittimo di Venezia. Il doge, imbarcatosi con il suo seguito sul Bucintoro, raggiungeva la bocca di porto di San Nicolò, dove il Patriarca benediva il mare; a suggello del matrimonio, il doge gettava in acqua un anello d'oro. Secondo la tradizione, la festività venne istituita attorno all'anno 1000 per celebrare la conquista della Dalmazia, ma fu nel 1177 che essa assunse carattere sacrale, quando papa Alessandro III, volendo ricompensare Venezia dei suoi servigi nel conflitto contro il Barbarossa, avrebbe proclamato l'egemonia della Repubblica sull'Adriatico.

(62) Cfr. Ph. Renaud, Lecture d'Apollinaire, cit., p. 175 .

(63) Cfr. n. 5 di questo saggio.

(64) Così recitava la Chanson du mal-aimé stampata nel 1909 sul «Mercure de France». La rielaborazione di questo verso, passato nel 1912 , in vista di
Alcools, al più celebre «triste et melodieux délire», è stata letta come un congedo dalla rassegnazione e dal gusto simbolisti: cfr. M. Richter, Apollinaire. Il rinnovamento della scrittura poetica all'inizio del Novecento, Il Mulino, Bologna, 1990, p. 17.

(65) La Tortue, v. 2, in G. ApollinAIRE, Euvres poétiques, texte établi et annoté par M. AdEma et M. DÉCAUDIN, Paris, Gallimard, 1956, p. 4. La prima edizione del Bestiaire, o Cortège d'Orphée, è del 1911, ma già in precedenza, nel 1908, Apollinaire aveva pubblicato questa e altre poesie della raccolta su «La Phalange», con il titolo di La marchande des quatre saisons ou le bestiaire mondain. La rima lyre-delire che ricorre nella Tortue (vv. 1-2) sta ad indicare un indirizzo preciso del fare poetico, e cioè il canto mitico e divino di Orfeo. Nella Chanson du mal-aimé la stessa rima (vv. 271-273) rivelerà al poeta la sua vocazione di creatore, sancendone la prima, sofferta vittoria. Mi pemetto di rinviare, a questo proposito, alle mie conclusioni in A. METLICA, Il primo orfismo. Il poeta secondo Apollinaire all'altezza del "Bestiaire", «Studi Francesi», 154, LII-1, 2008, pp. 136-146. 
dell'esprit nouveau; al di là di ogni sforzo sistematico, Apollinaire serba la disinvoltura e l'intelligenza di un dilettante di stendhaliana memoria ${ }^{66}$.

Aveva ragione Louis Aragon quando, nella préface ad una ristampa delle Onze mille verges ${ }^{67}$, instaurava un audace collegamento tra l'Apollinaire "serio", il lirico per cui la poesia «est avant tout une curiosité de l'inconnaissable» ${ }^{68}$, e l'Apollinaire erotico, curioso di versi di second rayon. E se, sulla scorta di quanto detto sin qui, si può rimproverare ad Aragon di aver esagerato, affermando che Apollinaire «prit à la traduction de Baffo le secret de l'accent d'un grand nombre de ses poèmes», non si può che ammirarne l'intuito quando attribuisce al nostro poeta «une conscience aussi claire des liens de la poésie et de la sexualité, une conscience de profanateur et de prophète» ${ }^{69}$.

ALESSANDRO METLICA

(66) Tra i due Apollinaire, l'editore e il poeta, non vi è dunque contraddizione, come ha mostrato L. CAmpa, Sous le signe d'Aphrodite, cit., p. 204: Apollinaire è sì «l'héritier d'une tradition éditoriale érudite née au XIX ${ }^{\mathrm{e}}$ siècle» ma, al contempo, «il utilise les interstices du genre dans lequel il s'exerce pour faire valoir ses idées».

(67) G. Apollinaire, Les onze mille verges ou les amours d'un hospodar, Monte-Carlo, Les Ygrées, 1930. Questa edizione, uscita per le cure di René Bonnel, presentava un'introduzione anonima, datata «mai 1930», che ora sappiamo essere di Louis Aragon (cfr. ID., Euvres en prose, cit., vol. III, pp. 1319-1320).

(68) Ivi, p. 1319.

(69) Ivi, p. 1320. 